\title{
Buffer gas cooling of ion beams
}

\author{
A. Kellerbauer*), T. Kim, R. B. Moore, P. Varfalvy \\ Foster Radiation Laboratory, Department of Physics, McGill University, \\ Montréal (Québec) H3A 2T8, Canada
}

\begin{abstract}
The cooling action of a buffer gas on ions contained within it can be used to cool an ion beam, thereby greatly improving its emittance and energy spread. It can also be used to greatly enhance the collection of an ion beam in an electromagnetic trap. The basic principles will be introduced in the context of a prototype system for such a beam cooler.
\end{abstract}

(Accepted for publication in Nucl. Instrum. Methods A)

*) Corresponding author. Current address: CERN, 1211 Genève 23, Switzerland. Telephone +41-22-7672780, facsimile +41-22-7678990, e-mail <a.kellerbauer@cern.ch> 


\section{Introduction}

The buffer gas cooling of ion beams is a subject of considerable recent interest. This is because of the realization by Kim and coworkers [1] that a low-pressure gas, usually referred to as a "buffer gas," can be used for cooling ion beams to close to the temperature of the gas itself.

The manipulation of ions in low pressure gases is a very old subject, dating back to the nineteenth century. However, manipulations that made use of the cooling properties of the gas, i.e. the tendency of the ion temperature to approach that of the colliding gas molecules, were hampered by the diffusion associated with such cooling. Without containment of the ions they simply become lost within the gas. Useful damping (i.e. "cooling") of ion motion by buffer gas was therefore only possible once ion confinement by electromagnetic fields became feasible in a gas.

Such containment was first proven to be feasible by Paul and coworkers [2].

Buffer gas damping of ion motion in a trap was first demonstrated by Hugget and Menasian in 1965 for $\mathrm{Hg}^{+}$ions in a Paul trap into which a small amount of helium was introduced. An introduction to the basic principles was presented by Dehmelt in 1967 [3]. A more thorough summary of the early work is given in the review book by Dawson [4]. Damping time constants achieved in such devices range typically from about 1 to $100 \mathrm{~ms}$.

A similar phenomenon has recently been demonstrated by Savard et al. [5] for the cooling of ion motion in a Penning trap. In this particular case, as observed by Penning, simple cooling of the magnetron motion would result in an expansion of the magnetron orbit, due to the fact that the overall energy of magnetron motion decreases with radius. However, in that work, the magnetron orbit was collapsed by using an azimuthal RF quadrupole field to transform its motion into cyclotron motion which does reduce in radius with reduced energy. This technique has now become very important in the preparation of ion collections for high-precision mass spectroscopy on Penning traps. Damping time constants achieved by this technique are typically about $100 \mathrm{~ms}$.

The application of RF quadrupole confinement to ion beams in a background of buffer gas was first demonstrated by Teloy and Gerlich at Freiberg in 1974 [6].

Subsequently Okuno [7] applied the technique to studies in physics. This was followed by the study of Douglas and French [8] of the effect of background gas on the transverse motion of ions in a radiofrequency quadrupole rod structure such as used in a quadrupole mass filter for chemical analysis. Indeed it was shown that background gas at moderate pressures, up to about $1 \mathrm{~Pa}$, did result in a significant increase in the transmission of ions through a 1-mm diameter orifice following the quadrupole rods.

These favours were returned to physics in the work of $\mathrm{Xu}$ and coworkers [9] on the transport of radioactive nuclei into cells for nuclear studies.

These results led to a study of the beam cooling process itself by Kim, cited above [1], which showed that a transverse radiofrequency quadrupole field could be used to confine ions injected at up to $100 \mathrm{eV}$ and subsequently cooled to close to room temperature by a buffer gas at pressures up to $100 \mathrm{~Pa}$. That work led to the work reported here.

The benefits of beam cooling derive from the concentration in phase space (the six-dimensional space made up of the momentum and position coordinates) that cooling of a collection of particles implies. Essentially, the smaller the volume occupied by the particles and the smaller the range of their momenta, the easier it is to detect them and to carry out precise measurements on them. In high-energy experiments, the cooling is achieved by immersing the beam in a bath of cold electrons running along with the beam at the average velocity of its particles. However, because of the relatively low velocities 
requirements on the trap and/or the extraction system. The operating principles of the cooler will therefore be presented in more detail.

\section{$2 \quad$ The cooling ion guide}

The radial constraint of a beam of ions that are being cooled by a buffer gas is most easily achieved by an axiperiodic oscillating electric quadrupole field. The confining properties of such a field were first presented in 1958 by Paul [2] and are now widely used in what are commonly called "quadrupole mass filters." In their application to ion beam confinement, the devices are run in a non-mass-selective mode and are referred to as "ion guides." The basic principles of such containment are available in many publications (see, for example, [4]) and will not be duplicated here. Only the pertinent features of the resulting motion within such a containment device will be presented.

Representative motions of an ion in an axiperiodic oscillating quadrupole electric field with buffer gas are shown in the computer simulations in Fig. 2, where the action of the buffer gas is treated as a simple viscous drag. At low pressures, the motion is seen to be basically oscillatory, as if the particles were contained within a simple harmonic potential well, with a small ripple at the frequency of the driving electric field. The effect of increased gas pressure is an increased damping of the oscillatory motion.

At a pressure of about $25 \mathrm{~Pa}$, the oscillatory motion is seen to be critically damped, leaving only the ripple caused by the driving field. At this point, the decay time of the excursion of an ion from the axis is minimal. In the case shown, where the gas is nitrogen, it is about $10 \mu \mathrm{s}$. For helium, the pressure at which this occurs will be about ten times higher. As the gas pressure is further increased, the decay time itself increases due to the increased viscosity.

Thus it is seen that buffer gas cooling of an ion beam can be very quick, of the order of several tens of microseconds. However, this high cooling rate comes at the expense of relatively high buffer gas pressures that could flood the vacuum systems of the incoming and exiting beams. The design of a practical system for using buffer gas cooling for an ion beam therefore requires a detailed study of the behavior of ions in an ion guide at various buffer gas pressures.

In order to conduct such a study, a radio-frequency quadrupole (RFQ) ion guide system was built for ${ }^{133} \mathrm{Cs}^{+}$ions in nitrogen. This combination was selected because of the relatively high buffer gas pressures that can be accommodated with nitrogen and because of the well-known interaction potentials for collisions between a cesium ion and a nitrogen molecule [14]. Thus the behavior of ions in the guide can be simulated by Monte-Carlo calculations, facilitating the interpretation of experimental results.

The ion guide for this system was made of four rods of diameter $16 \mathrm{~mm}$ and length $180 \mathrm{~mm}$, arranged as a quadrupole separated by $14 \mathrm{~mm}$ between opposite rods. The rods were housed in a container, itself inside a high-capacity $(2000 \mathrm{l} / \mathrm{s})$ pumping system. Orifices of $1 \mathrm{~mm}$ diameter were provided at each end of the container to allow the entrance and extraction of ion beams. With this system, buffer gas pressures beyond $100 \mathrm{~Pa}$ could be maintained while still allowing the injection of a $100-\mathrm{eV}$ beam of ${ }^{133} \mathrm{Cs}^{+}$ions into the gas and their extraction after cooling. By segmenting the rods into 5-mm disks and connecting the segments to a resistor chain, a relatively uniform DC electric field could be maintained along the axis of the structure. This allowed control of the axial as well as the radial motion of the ions.

A representative set of transmission curves obtained with this apparatus is shown in Fig. 3. It is seen that at a low buffer gas pressure, the ion transmission is only about $10 \%$. 

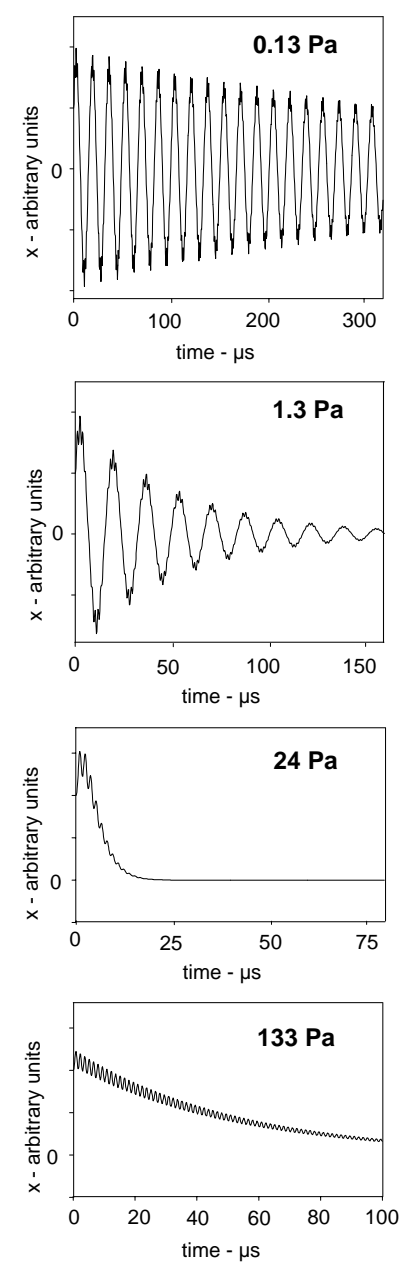

Figure 2: Representative motions of an ion in an axiperiodic oscillating electric quadrupole field with nitrogen as a buffer gas. The frequency of the field is $700 \mathrm{kHz}$. The ion is ${ }^{133} \mathrm{Cs}^{+}$ and the strength of the field is such that the macro-oscillation frequency, as can be seen in the figure, is about $50 \mathrm{kHz}$. 

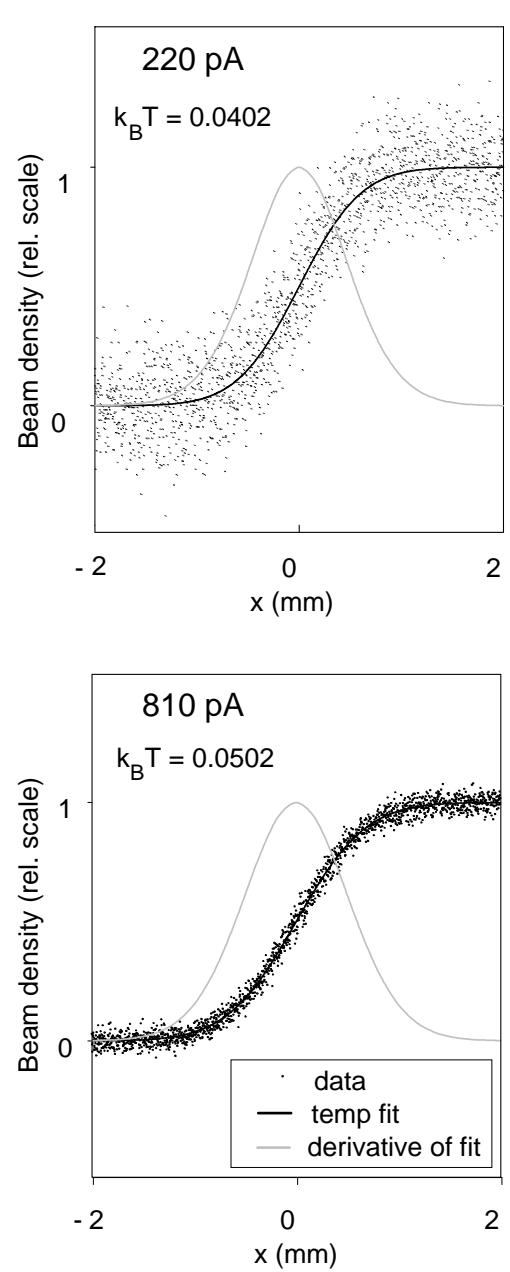

Figure 4: Typical beam profiles obtained by the rotating beam profiler.

was $3.5 \mathrm{~mm}$, so the RF potential amplitude of the vanes was set to $1 / 16$ th that of the main rods. Such a transition region allows the ions to escape from the buffer gas region into the extraction region without experiencing any change in the electric field that confines them. After exiting the vane structure, the ions were accelerated to a collection plate.

The profile of the extracted beam was obtained by making the collection plate from a pair of copper semicircles mounted on an insulating disk and rotated so that the gap between the plates crossed the extracted beam. Monitoring the current on the plates gave an integral of the beam profile. Figure 4 shows that the profiles obtained by this method were approximately Gaussian, as would be expected for the distribution of an ion collection that is in thermal equilibrium while it is being restrained within a simple harmonic potential well. The solid lines shown in Fig. 4 were obtained by fitting such distributions to the data, with the temperature as the only free parameter.

It is seen that the extracted beam is indeed about $2 \mathrm{~mm}$ wide, in keeping with the transmission of about $60 \%$ through a $1-\mathrm{mm}$ hole. (The actual diameter inside the ion guide is not exactly the same as at the collector due to the focusing effects of the extraction field. For details of how this is taken into account in the fits shown in Fig. 4, see [1].) It is seen from the fits that the ions in the beam are cooled to approximately twice the temperature of the buffer gas molecules. This is in keeping with the results of Lunney [15] for the temperature of ions cooled by buffer gas in a Paul trap. It is also seen 


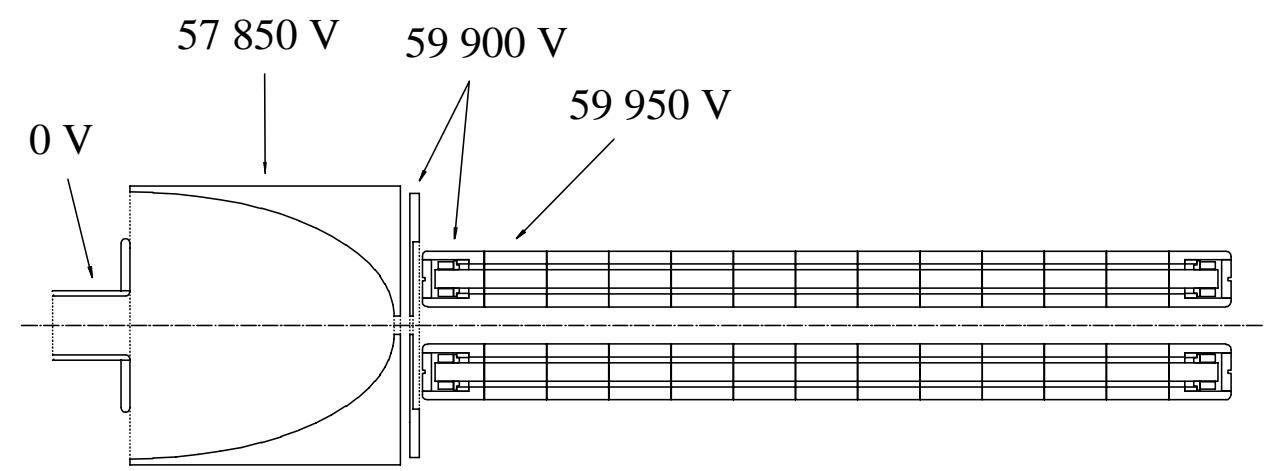

Figure 7: A cross-section of the decelerator-cooler system that was built as a test bed for the system being developed for the ISOLTRAP experiment. The potentials shown are for the deceleration and capture of a $60-\mathrm{keV}$ beam.

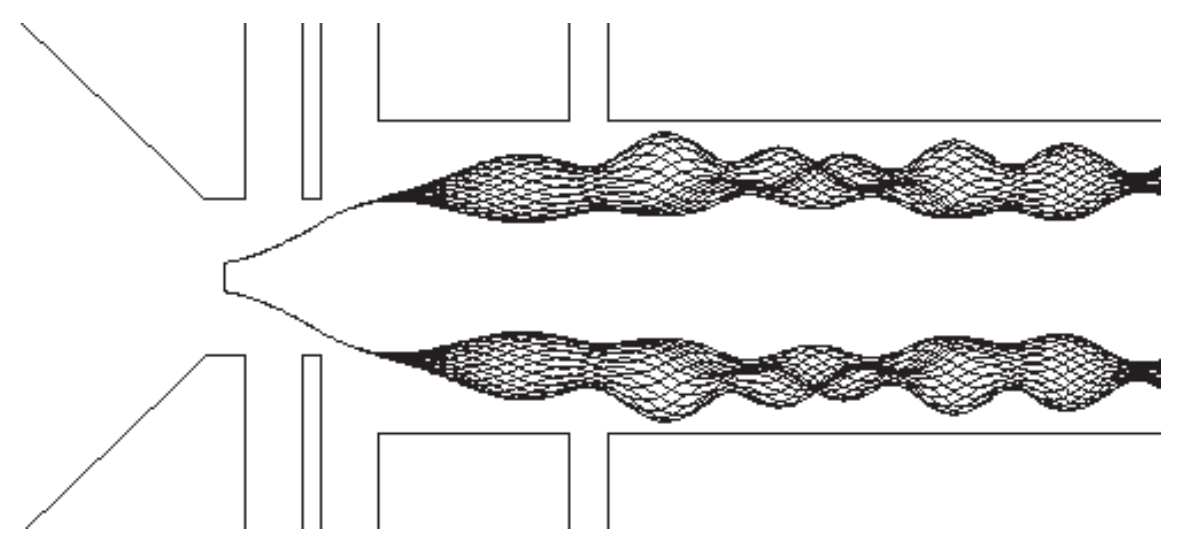

Figure 8: The calculated beam profiles for a typical ISOLDE ion beam injected into the RFQ ion guide for cooling. The different profiles are for the same ion beam entering at different RF phases. The start of the profile maps is at a beam energy of $1900 \mathrm{eV}$ after deceleration from $60 \mathrm{keV}$. The initial emittance of the $60-\mathrm{keV}$ beam is taken to be an ellipse of $30 \pi \cdot \mathrm{mm} \cdot \mathrm{mrad}$. The separation of the rods forming the ion guide is $12 \mathrm{~mm}$. The profiles are shown for a depth of $60 \mathrm{~mm}$ into the guide. 
regimes, the general requirement of clean, rounded, and polished surfaces applies for all electrodes, but there are specific considerations for each region. For the atmospheric part of the system, the size of the cages to be kept at high voltage requires safe shielding and safeguards against surge currents from the inevitable occasional spark-overs. Inside the vacuum system, the arc problems are ameliorated somewhat, particularly for electrodes that are at a positive potential relative to their surroundings so that the emission of electrons is prohibited. Also, for such electrodes, a slight gas pressure (about $10 \mathrm{mPa}$ seems to be optimal) can actually increase the breakdown potential, possibly due to the cleaning of the electrode surface by sputtering [17]. However, it appears that potential gradients should always be kept to less than about $5000 \mathrm{~V} / \mathrm{mm}$.

For the buffer gas region of the system, one is often in the regime where the breakdown potential in the gas is minimal [18]. This is of concern when trying to achieve the highest possible electric quadrupole potential on the rods of the ion guide. Here it is important to avoid long paths for electrons to follow in moving between electrodes since it is such long paths that allow the electron to encounter a gas molecule and ionize it.

Another important consideration is the cleanliness of the vacuum system. This is because of the many collisions with molecules that an ion will encounter during its cooling and hence the many opportunities for exchange of its charge and escape from the system. This is not so critical for a relatively benign ion such as ${ }^{133} \mathrm{Cs}^{+}$, but for more aggressive ions, particularly those of noble gases, one encounter with a contaminant molecule can lead to instant loss. The vacuum system should therefore be fabricated using polished stainless steel or oxygen-free copper and non-organic insulators as much as possible and should be designed to be bakeable. Only ultra pure sources of gas should be used as buffers.

Another design consideration that applies to any RF containment system is that all insulating surfaces should be kept very clear of the RF electric field. This is because of the possibility that stray electrons may be deposited on an insulator surface by the RF field and remain there as a DC patch of charge which can severely disturb the confinement properties of the RF field itself.

With proper attention to these considerations and with the experience gained using the prototype ion guide now in operation at McGill University as well as RFQ ion beam coolers in operation at radioactive-beam facilities, it should bepossible to reach overall collection and cooling efficiencies of close to $100 \%$ for ISOLDE-type beams. The energy spread of such beams after cooling should approach that in the work described here: twice room temperature or about $50 \mathrm{meV}$ (FWHM). The emittance that can be obtained with such cool beams will depend on the strength of confinement [19]. From the results presented here, it is expected that normalized emittances of the order of $0.5 \mathrm{eV} \cdot \mu \mathrm{s}$ should be achievable (corresponding to about $1 \pi \cdot \mathrm{mm} \cdot \mathrm{mrad}$ for a $60 \mathrm{keV}$ beam).

As for the ion current that can be cooled, the results presented here show that currents of up to $1 \mathrm{nA}$ display only a slight rise in beam temperature and hence a corresponding slight increase in emittance. This is no doubt due to the radial field of the space charge of the higher-current beam which pushes more ions out into a higher quadrupole field. This results in higher-energy collisions with the buffer gas molecules and hence in a higher temperature of the ion [15], an effect which is commonly called "RF heating." This effect will certainly get worse for larger currents but can be reduced somewhat by applying a greater axial field in the ion guide so that the beam is stretched. However, a high axial field can itself induce heating from an increase in collision energy due to the axial motion. The practical limit for the beam current that can be cooled will therefore 
depend on the emittance and energy spread that one is willing to tolerate. From general considerations, it is expected that it should be possible to cool currents of up to $1 \mu \mathrm{A}$ to an energy spread of less than $1 \mathrm{eV}$ and normalized emittances of less than $2 \mathrm{eV} \cdot \mu \mathrm{s}$.

The problems of high beam current in an ion guide could be ameliorated somewhat by deliberately introducing negative ions that would neutralize the space charge of the positive beam. This is possible in RF quadrupole confinement because both positive and negative ions are confined simultaneously. Furthermore, the micromotions of the two are out of phase so that the possibility is considerably reduced of their being at rest relative to each other and therefore subject to charge exchange. However, with the axial fields necessary to pull the cooled ions through the buffer gas, it may not be possible to sustain enough negative ions to have an appreciable space charge neutralization.

In any case, the primary use of beam cooling is expected to be with weak beams that must be collected and cooled to enable their detection. As pointed out above, it is very important to collect such beams into bunches in a trap. Here the important considerations are not only the nature of the collected bunch but also the time it takes to prepare such a bunch. From the work reported here, it is seen that such a bunch will take about $1 \mathrm{~ms}$ to be formed. As with the cooled beam, the transverse emittance will depend on the temperature of the ions and the degree of radial confinement within the trap. Likewise, the longitudinal emittance of the bunch will depend on the ion temperature and the strength of the axial confinement.

However, such a trap will not be strong enough to contain many ions without excessive heating due to their space charge repulsion. It is expected that the limit will be of the order of several thousand and very modest beam currents will therefore overload the trap very quickly. This can be a problem if it is largely made up of contaminant isobaric ions from which the desired ions must be separated. For such cases it may well be appropriate to have the cooled ion beam collected in a large Penning trap immediately at the end of the cooler. This is possible because the magnetic field of the Penning trap would have a negligible effect on the RFQ ion guide itself. Although this would be considerably more expensive than a small linear incorporated in the RFQ ion beam cooler, it would allow the continuous stream of ions to be accumulated directly.

We hope that this contribution shows that 40 years after the development of the first quadrupole ion traps, buffer gas cooling finally offers the possibility of collecting any beam of rare ions and holding them in an electromagnetic trap for careful study. The authors are convinced that these advances will significantly enhance precision studies with radioactive nuclear beams all over the world.

\section{Acknowledgments}

This work was funded by the Natural Sciences and Engineering Research Council of Canada.

\section{References}

[1] T. Kim. Buffer gas cooling of ions in a radio frequency quadrupole ion guide. Ph. D. thesis, McGill University, 1997

[2] W. Paul, H. P. Reinhard, and U. v. Zahn, Z. Phys. 152 (1958) 143.

[3] H. G. Dehmelt, Adv. At. Mol. Opt. Phys. 3 (1967) 53.

[4] P. H. Dawson, ed. Quadrupole Mass Spectrometry and its Applications. New York: American Institute of Physics, 1995.

[5] G. Savard et al., Phys. Lett. A 158 (1991) 247. 
[6] E. Teloy and D. Gerlich, Chem. Phys. 4 (1974) 417.

[7] K. Okuno, J. Phys. Soc. Jpn. 55 (1986) 1504.

[8] D. J. Douglas and J. B. French, J. Am. Soc. Mass Spectrom. 3 (1992) 398.

[9] H. J. Xu et al., Nucl. Instrum. Methods A 333 (1993) 274.

[10] G. Bollen et al., Nucl. Instrum. Methods A 368 (1996) 675.

[11] G. Savard et al., proceedings of the APAC2000 conference, Cargese, September 2000, to appear in Hyperfine Interactions.

[12] A. Jokinen et al., proceedings of the RNB2000 conference, Divonne, April 2000, to appear in Nucl. Phys.

[13] J. Dilling, proceedings of the APAC1999 conference, September 1999, Mainz, Hyperfine interactions 127 (2000) 491.

[14] E. A. Mason and E. W. McDaniel. Transport Properties of Ions in Gases. New York: John Wiley \& Sons, 1988

[15] M. D. N. Lunney. The Phase Space Volume of Ion Clouds in Paul Traps. Ph. D. thesis, McGill University, 1992

[16] A. Kellerbauer. Production of a cooled ion beam by manipulation of 60-keV ions into a radio-frequency quadrupole ion guide. M. Sc. thesis, McGill University, January 1999.

[17] R. V. Latham. High Voltage Vacuum Insulation. New York: Academic Press, 1995

[18] Y. P. Raizer. Gas Discharge Physics. Berlin: Springer-Verlag, 1991

[19] A. M. Ghalambor Dezfuli. Injection, Cooling and Extraction of Ions from a Very Large Paul Trap. Ph. D. thesis, McGill University, 1996 